\title{
VACUUM BEAM LOSSES IN THE AXIAL INJECTION SYSTEMS OF THE FLNR HEAVY ION CYCLOTRONS
}

\author{
M.N. El-Shazly, G.G. Gulbekian, A.I. Ivanenko, M.V. Khabarov, A.V.Tikhomirov \\ FLNR， Joint Institute for Nuclear Research, Dubna, Moscow region, 141980 Russia
}

\begin{abstract}
A simulation program was carried out to calculate the pressure distribution and the beam loss due to the charge exchange cross section in order to optimize the vacuum system parameters of the heavy ion transmission lines. A series of measurements was set up to evaluate the beam loss in the axial injection systems of the FLNR cyclotrons. The pressure measurements were compared with the program.
\end{abstract}

\section{INTRODUCTION}

The isochronous cyclotrons U-400M and U-400 [1] have been equipped with ECR ion sources provided with axial injection systems [2]. They were located atop the cyclotron magnets [3,4]. This paper presents a model for calculating the pressure distribution and the beam losses due to the charge exchange with the residual gasses in the axial injection system. The result of the calculations made are compared with those of some pressure measurements. Also Investigated the manner in which the heavy ion beam transmission factor of the ion guide pipe depends on the following parameters: the diameters of its parts, the pumping speed, the gas loading from the ECR ion source, and the gas desorption rate.

\section{VACUUM SYSTEM}

In any vacuum system, the first important gas source is formed by the static or thermal outgassing of weakly adsorbed molecules and diffusion of gasses. The systems concerned have specific static outgassing rate ranging from $1.3 \times 10^{-7}$ to $1.3 \times 10^{-6} \mathrm{~Pa} \ell / \mathrm{sec} / \mathrm{cm}^{2}$ depending on the pumping time. Responsible for the second important gas source is the dynamic outgassing in the presence of the beam, which results from two causes. The first cause is accounted by the gas loading from the ECR ion source. The second cause lies in strongly adsorbed molecules being released because of the lost ion beam striking the ion guide pipe.

\section{PRESSURE DISTRIBUTION}

A simulation program (GENAP7) was developed estimating the pressure distribution through the ion guide pipe and the ion beam transmission efficiency in relation to the pressure distribution pattern. The program proposed is capable of calculating the pressure distribution through the ion guide pipe comprising any number of parts of various forms and gas molecular conductivity. Within the molecular flow regime the flow of molecules through the ion guide pipe is expressed by the equations

$Q(x)=-w \frac{d p}{d x} \quad$ and $\quad \frac{d Q}{d x}=-A q$

Where

$Q(\mathrm{~Pa} \ell / \mathrm{sec}) \quad$ gas flow.

$A\left(\mathrm{~cm}^{2} / \mathrm{m}\right) \quad$ specific surface area .

$q\left(\mathrm{~Pa} \ell / \mathrm{sec} / \mathrm{cm}^{2}\right)$ specific static outgassing rate.

$p(\mathrm{~Pa}) \quad$ pressure.

$w(\mathrm{~m} \ell / \mathrm{sec}) \quad$ specific molecular conductance.

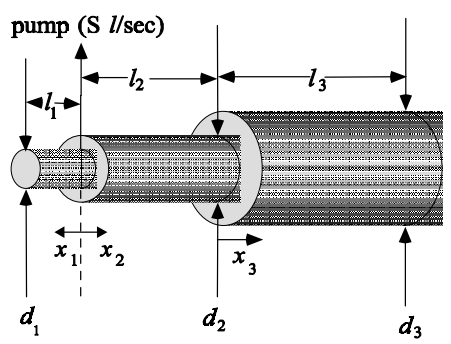

Figure 1.

To explain the mathematical algorithm of the program let us divide our vacuum chamber into three parts each part being of length $l_{i}$, and diameter $d_{i}$. The specific static outgassing in the $i$-part will be $q_{i}, i=1,2,3$ (see Fig. 1). Granted that there is only one pump and no beam, just only the static outgassing, the pressure distribution along the coordinates $x_{1}, x_{2}$, and $x_{3}$ is defined by the equations [5].

$$
\begin{array}{r}
P_{q}\left(x_{1}\right)=\frac{q_{1} \pi d_{1} l_{1}}{S}+\frac{q_{2} \pi d_{2} l_{2}}{S}+\frac{q_{3} \pi d_{3} l_{3}}{S}+ \\
\frac{q_{1} \pi d_{1} x_{1}}{12 d_{1}^{3}}\left(l_{1}-\frac{x_{1}}{2}\right) \\
P_{q}\left(x_{2}\right)=\frac{q_{1} \pi d_{1} l_{1}}{S}+\frac{q_{2} \pi d_{2} l_{2}}{S}+\frac{q_{3} \pi d_{3} l_{3}}{S}+ \\
\frac{q_{3} \pi d_{3} l_{3} x_{2}}{12 d_{2}^{3}}+\frac{q_{2} \pi d_{2} x_{2}}{12 d_{2}^{3}}\left(l_{2}-\frac{x_{2}}{2}\right) \\
P_{q}\left(x_{3}\right)=\frac{q_{1} \pi d_{1} l_{1}}{S}+\frac{q_{2} \pi d_{2} l_{2}}{S}+\frac{q_{3} \pi d_{3} l_{3}}{S}+ \\
\frac{q_{3} \pi d_{3} l_{3} l_{2}}{12 d_{2}^{3}}+\frac{q_{2} \pi d_{2} l_{2}^{2}}{24 d_{2}^{3}}+\frac{q_{3} \pi d_{3} x_{3}}{12 d_{3}^{3}}\left(l_{3}-\frac{x_{3}}{2}\right)
\end{array}
$$


$\mathrm{S}(\ell / \mathrm{sec}) \quad$ is the pump speed

The gas desorption rate due to the lost beam was calculated according to the next equation [6].

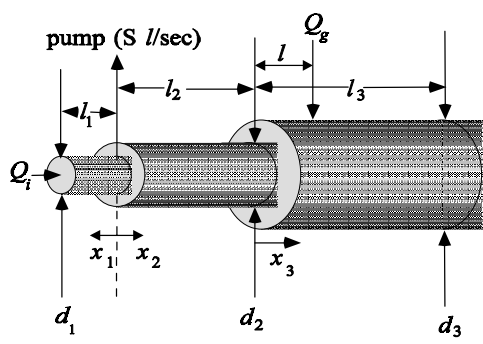

Figure 2.

$$
Q_{g}=1000 \frac{I}{Z} \gamma_{i} K T
$$

Where

$Q_{g}(\mathrm{~Pa} \ell / \mathrm{sec})$ the gas desorption rate due to lost beam $I$ (A) the lost beam intensity

$Z$ (coulomb) the average charge state of the lost ions

$\gamma_{i}$ (mol./ion) the gas desorption yield

$K\left(\right.$ Joule $\left./{ }^{\circ} \mathrm{K}\right) \quad$ Boltzmann constant

$T\left({ }^{\mathrm{O}} \mathrm{K}\right) \quad$ gas temperature

Granted that the system configured as shown in Fig. 2 is only of one pump and static outgassing-free, the gas loading from the ion source being $Q_{i}$ (is expected to range from $3 \times 10^{-3}$ to $1.6 \times 10^{-2} \mathrm{~Pa} \ell / \mathrm{sec}$ ) and the gas desorption due to beam losses $Q_{\mathrm{g}}$, then the pressure distribution through the vacuum chamber concerned will be as follows:

$$
\begin{aligned}
& P_{Q}\left(x_{1}\right)=\frac{Q_{g}}{S}+Q_{i}\left(\frac{x_{1}}{12 d_{1}^{3}}+\frac{1}{S}\right) \\
& P_{Q}\left(x_{2}\right)=\frac{Q_{i}}{S}+Q_{g}\left(\frac{x_{2}}{12 d_{2}^{3}}+\frac{1}{S}\right) \\
& P_{Q}\left(x_{3}\right)=\frac{Q_{i}}{S}+Q_{g}\left(\frac{l_{2}}{12 d_{2}^{3}}+\frac{1}{S}+\frac{x_{3}}{12 d_{3}^{3}}\right) \text { if } x<l \\
& P_{Q}\left(x_{3}\right)=\frac{Q_{i}}{S}+Q_{g}\left(\frac{l_{2}}{12 d_{2}^{3}}+\frac{1}{S}+\frac{l}{12 d_{3}^{3}}\right) \text { if } x>l
\end{aligned}
$$

If there is in addition static outgassing the pressure distribution is founded by combining equations (2) and (4).

$$
P_{j}\left(x_{i}\right)=P_{q}\left(x_{i}\right)+P_{Q}\left(x_{i}\right)
$$

To return to the axial injection systems of the U-400 and U-400M cyclotrons, there are more than one pump located at four sites along the length of each ion guide pipe. An iteration process is performed to obtain the pressure distribution along the ion guide pipe. The first iteration is according to the next equation:

$$
\frac{1}{P\left(x_{i}\right)}=\sum_{j=1,4} \frac{1}{P_{j}\left(x_{i}\right)}
$$

Each pump in the system is responsible for pumping the part of the ion guide pipe that is located between the nearest maximum pressure points. The iteration process will continue until the coincidence of the maximum pressure points will be obtained.

The pressure was measured at three points in each system at various beam current intensities and varying the number of pumps in operation. The results of the measurements were compared with those of the simulations. The results of the simulation and measurements are shown in Figs. 3 and 4.

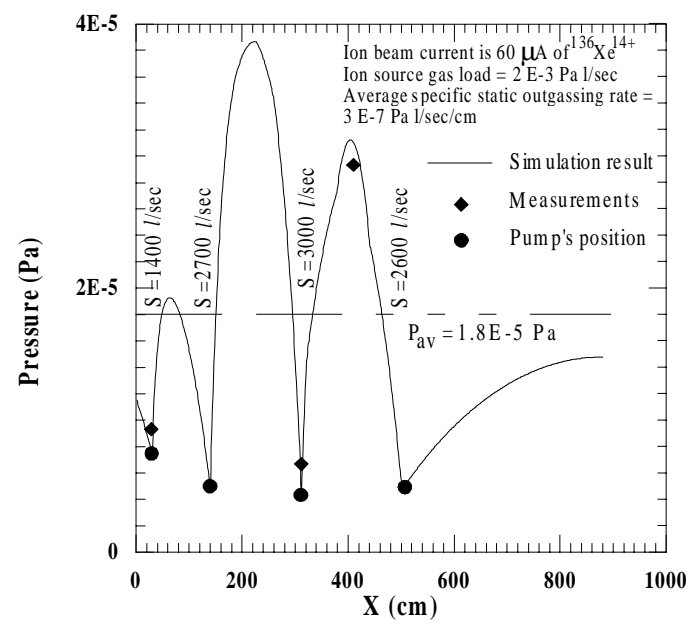

Figure 3: The pressure distribution in the ion guide pipe of the axial injection system of the U-400 cyclotron.

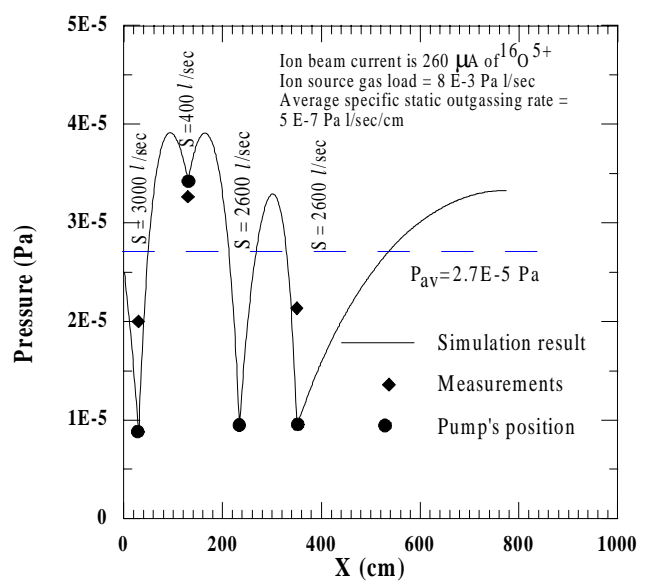

Figure 4: The pressure distribution in the ion guide pipe of the axial injection system of the U-400M cyclotron.

\section{TRANSMISSION FACTOR}

The transmission factor depends on the pressure distribution and the charge exchange cross section between the ion beam and the residual gas. The pressure distribution can be founded as indicated above. The ECR ion source injection voltage lies $12-25 \mathrm{kV}$ range depending on the ion beam charge-to-mass ratio. In this energy range, the single-electron capture cross section is much higher than that of single-electron loss cross section 
and of multi-electron capture cross section. An approximate scaling rule can be used for this energy range [7].

$\sigma_{z, z-1}=1.43 \times 10^{-12} z^{1.17} R^{-2.76}$

where

$\sigma_{\mathrm{Z}, \mathrm{Z}-1}\left(\mathrm{~cm}^{2}\right)$ the electron capture cross section

$z$ (coulomb) the ion (projectile) charge

$R$ (Joule) the first ionization potential of the target (residual gas).

In this case, the transmission factor for any ion in the ion guide pipe can be obtained easily using the next formula :-

$$
\tau=\exp \left(-2.69 \times 10^{14} \sigma_{z, z-1} \int_{0}^{L} P\left(x_{i}\right) d x_{i}\right)
$$

where

$$
\begin{array}{ll}
\tau & \text { transmission factor } \\
\sigma_{\mathrm{Z}, \mathrm{z}-1}\left(\mathrm{~cm}^{2}\right) & \text { the single electron capture cross section } \\
P(\mathrm{~Pa}) & \text { pressure }
\end{array}
$$

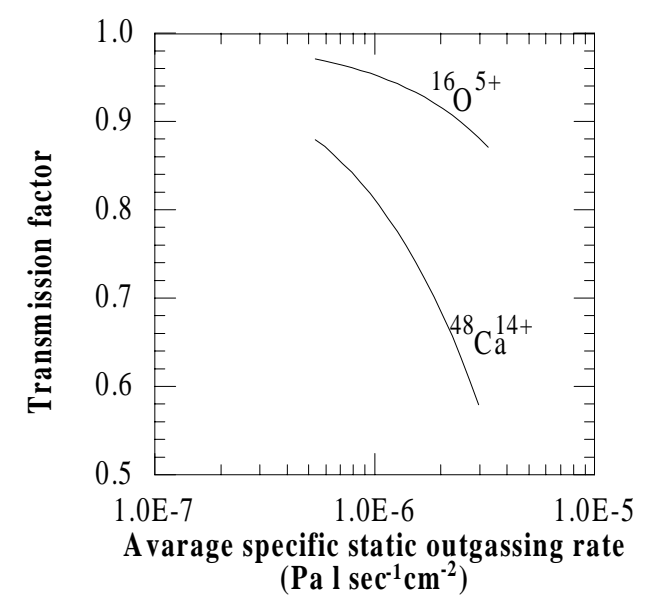

Figure 5: The specific static outgassing effect on the transmission factor through the axial injection system of the U-400M cyclotron.

The transmission factor is seen to depend on the average pressure only. On this basis, the relationships between the transmission factors and the vacuum system's parameters were investigated under the conditions that are written in Figs. 3 and 4. For example, in the axial injection system of the U-400M cyclotron, where ${ }^{16} \mathrm{O}^{5+}$ and ${ }^{48} \mathrm{Ca}^{14+} 260 \mu \mathrm{A}$ beams were being considered and the gas loading from the ion source was being taken to be about $8 \times 10^{-3} \mathrm{~Pa} \ell / \mathrm{sec}$, the relation between the transmission factor and the specific static outgassig rate is presented in Fig. 5. The diameter dependence of the transmission factor in the axial injection system of the U-400 cyclotron is shown in Fig. 6.

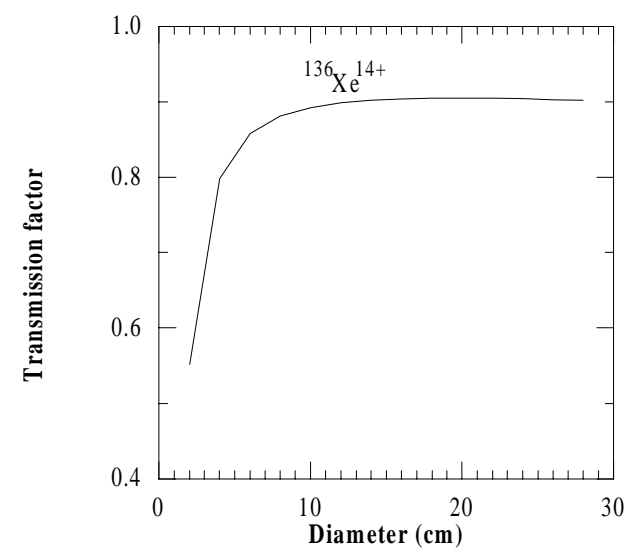

Figure 6: The transmission factor due to the variation of the diameter of the ion guide pipe in the axial hole of the U400 cyclotron magnet.

\section{CONCLUSION}

The investigation of various parameters affecting the pressure distribution and the transmission factor of the axial injection systems of the U-400 and U-400M cyclotrons shows that:

- The most important parameter is the static outgassing.

- The pumping speed is an important parameter from the stand point of reaching the required level of the static outgassing as quickly as possible.

- The transmission factor increases insignificantly with the diameters of the ion guide pipe for the values of the diameters greater than those of the existing configuration.

- It is possible to increase the ion beam current up to $1 \mathrm{~mA}$ and the beam losses will not be much higher.

The simulation program is useful to optimize the vacuum parameters of the transmission lines and linear accelerator after chosing the cross section formula in their range of energies.

\section{REFERENCES}

1. Gulbekian G. et al., 13th Inter. Conf. on Cyclotrons and Their Application, Vancouver, 1992, p.11

2. Efremov A. et al., Rev. Sci. Inst., V. 65 (4), 1994, p.1084.

3. Gulbekian G. et al., Sci. Rep. 1993-1994, FLNR, JINR, P. 227.

4. Yu.Ts. Oganessian et al., Sci. Rep. 1995-1996, FLNR, JINR, P. 270.

5. Kalinin Yu Zh. et. al., Vacuum, V. 46, No. 7, 1995, p.717.

6. Gröbner O, CAS, CERN, V. 2, 85-19, 27 Nov. 1985, p489.

7. Müller A. et al., Inst. Phys. Conf. , No. 38, 1978, Chapter 4. 